\title{
Correction to "In Vitro Assessment of Synthetic Nano Engineered Graft Designed for Further Clinical Study in Nerve Regeneration"
}

\author{
Ali Sadeghi ${ }^{\circledR}$, Fatholah Moztarzadeh ${ }^{1 * \mathbb{(}}$, Jamshid Aghazadeh Mohandesi², Claudia \\ Grothe $^{3}$, Kirsten Haastert Talini' ${ }^{3}$, Ali Reza Zalli ${ }^{\circledR}$, Reza Jalili Khoshnoud ${ }^{4}$ \\ ${ }^{1}$ Faculty of Biomaterial and Tissue Engineering, Department of Biomedical Engineering (Center of \\ Excellence), Amirkabir University of Technology, Tehran, Iran \\ ${ }^{2}$ Department of Material Science \& Engineering, Amirkabir University of Technology, Tehran, Iran \\ ${ }^{3}$ Institute of Neuroanatomy, Hannover Medical School, Hannover, Germany and Center for Systems \\ Neuroscience (ZSN), Hannover, Germany \\ ${ }^{4}$ Neurofunctional Research Center, Shohada Tajrish Neurosurgical Comprehensive Center of \\ Excellence, Shahid Beheshti University of Medical Science, Tehran, Iran
}

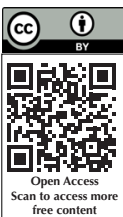

*Correspondence to Fatholah Moztarzadeh, Email: moztarzadeh@aut.ac.ir

Published online June 30, 2021

I $\mathrm{n}$ the article entitled "In vitro assessment of synthetic nano engineered graft designed for further clinical study in nerve regeneration" which published in International Clinical Neuroscience Journal 2018;5(3)8691, Figure 6 contained some errors, in which panels A, $\mathrm{C}$, and D have been reported mistakenly. This mistake happened inadvertently during the data processing and data collection. The corrected Figure 6 and corresponding caption is presented in the present correction, in which the correct SEM images replaces in panels A, C, and D. The reader should note that this error does not affect the scientific quality of the work and no other section of the manuscript is affected by this error. In addition, the correction does not affect the conclusions of that paper. We would like to apologise for any inconvenience caused.

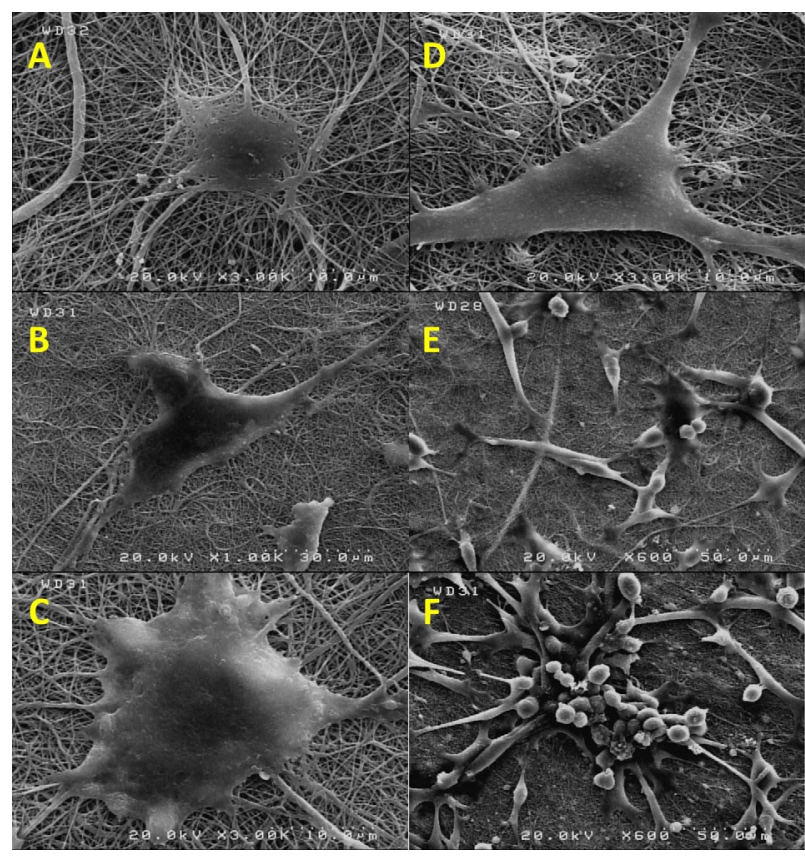

Figure 6. SEM Images of PC12 Seeded on the Nanofibrous Scaffold. (A) PCL in Chloroform, (B) PCL in DMF, (C) PCL in Acetic/Formic Acid, (D) Surface Modified PCL With Plasma, (E) PCL/Gelatin, (F) PCL/Chitosan. 